\title{
Factors affecting successful use of intranasal dexmedetomidine: a cohort study from a national paediatrics tertiary centre
}

\author{
Lijia Fan ${ }^{1}$, Yinghao Lim², Gloria Songmei Wong', Ryan Taylor ${ }^{1}$ \\ ${ }^{1}$ Department of Paediatrics, Khoo Teck Puat-National University Children's Medical Institute, National University Hospital, Singapore, Singapore; \\ ${ }^{2}$ Department of Cardiology, National University Heart Centre, Singapore, Singapore \\ Contributions: (I) Conception and design: L Fan, R Taylor; (II) Administrative support: GS Wong; (III) Provision of study materials or patients: None; \\ (IV) Collection and assembly of data: GS Wong, L Fan; (V) Data analysis and interpretation: L Fan, Y Lim; (VI) Manuscript writing: All authors; \\ (VII) Final approval of manuscript: All authors. \\ Correspondence to: Dr. Lijia Fan, MBBS, MMed (Paeds), MRCPCH (UK). Department of Paediatrics, NUHS Tower Block, Level 12, 1E Kent Ridge \\ Road, Singapore 119228. Email: li_jia_fan@nuhs.edu.sg.
}

Background: Use of intranasal (IN) dexmedetomidine for procedural sedation has been reported in recent
years. Good patient selection is important to ensure high success rates. We aimed to identify factors that
influence the successful use of IN dexmedetomidine in non-invasive investigations.
Methods: All paediatric patients who received IN dexmedetomidine for investigations between 01 July
2019 to 01 July 2020 were included. Baseline demographics, time to reach adequate sedation level, duration
of sedation, dose, indications for sedation and need for rescue sedatives were recorded. Procedures were
classified into "long" or "short" according to completion time. Successful sedation was defined by completion
of investigations by IN dexmedetomidine alone.

Results: Of 105 patients included, median age was 20.0 months, and median weight $11.0 \mathrm{~kg}$. Magnetic resonance imaging $(56,53.3 \%)$ was the most common indication. Sixty $(57.1 \%)$ were successfully sedated using IN dexmedetomidine alone. Automated auditory brainstem response, computerised tomography and mercaptoacetyltriglycine-3 renogram scans had the highest success rate $(83.3 \%, 83.3 \%$, and $100 \%$ respectively). On multivariate analysis, short procedures had an adjusted odds ratio of 5.30 (95\% CI: $1.69-$ 16.61; $\mathrm{P}=0.004)$ compared to long procedures.

Conclusions: IN dexmedetomidine is effective for procedural sedation for paediatric patients. The most important predictor for sedation success was indication of sedation and duration of procedures.

Keywords: Paediatric; sedation; intranasal (IN); dexmedetomidine

Submitted Oct 31, 2020. Accepted for publication Feb 15, 2021.

doi: $10.21037 /$ tp-20-358

View this article at: http://dx.doi.org/10.21037/tp-20-358

\section{Introduction}

Dexmedetomidine is a selective alpha-2 adrenoceptor agonist with activity mainly in the central nervous system. It has been well established as an anxiolytic and sedative during the perioperative period and in the intensive care unit (ICU) but not in procedural sedation (1). In recent years, the use of intranasal (IN) dexmedetomidine as a procedural sedative for automated auditory brainstem response (AABR), magnetic resonance imaging (MRI) and computerised tomography (CT) has also been reported (2-6). When compared to oral chloral hydrate, it has been shown to be more efficient in achieving a satisfactory sedative state when conducting AABR (7).

There are growing concerns for traditional sedatives. The use of oral chloral hydrate has been banned in Italy and France due to potential carcinogenicity $(8,9)$. In addition, potential neurotoxicity of traditional intravenous (IV) sedatives such as benzodiazepines, and barbiturates have also been reported (10). On the other hand, dexmedetomidine 
Table 1 Modified Ramsay scale

Awake State
1. Anxious
2. Cooperative, oriented, tranquil
3. Asleep, brisk response to loud auditory stimulus
Sleep state
4. Asleep, sluggish response to loud auditory stimulus
5. No response to loud auditory stimulus
6. Does not response to painful stimulus

has shown evidence of a neuroprotective effect in animal studies $(11,12)$.

Despite the above, use of IN dexmedetomidine has not been established as a standard. Criticism of prior studies are twofold: firstly, the successful use of IN dexmedetomidine was defined by the ability to reach an adequate sedation level, or by achieving successful parental separation $(3,5)$. Few papers have examined the more clinically relevant outcome of successful completion of procedures or investigations. Secondly, most studies are limited to a single indication for the use of IN dexmedetomidine, and few have reported more generalised use across various painless procedures and investigations.

In this study, we aimed to investigate the use of IN dexmedetomidine as a solo sedative and successful completion of non-invasive procedures in a national paediatric tertiary centre and analyse factors that can affect the outcome.

We present the study in accordance with the STROBE reporting checklist (available at http://dx.doi.org/10.21037/ tp-20-358).

\section{Methods}

The study was a retrospective cohort study of paediatric patients who had received IN dexmedetomidine for procedural sedation for the period from 01 July 2019 to 01 July 2020 in a national paediatrics tertiary hospital in Singapore. Inclusion criteria were age between 1 month to 18 years, and sedation using IN dexmedetomidine by the paediatric sedation service. Exclusion criteria were sedations for invasive procedures such as peripherally inserted central line insertion and lumbar punctures, terminated procedures or investigations for reasons not related to sedation, and allergy to dexmedetomidine.

All sedations were accompanied by the paediatric sedation team which was made up of a sedation qualified specialist nurse as well as a paediatric intensive care physician. Patients were reviewed by the physician before the procedure and an IV cannula placed. Six intensive care physicians participated in the study. The choice of sedatives was left to the discretion of the physician. Factors which can influence the physician's decision include estimated duration of the procedure and previous sedation history. Available sedatives included IV propofol, midazolam, ketamine and oral chloral hydrate. IN dexmedetomidine was administered at a dose of 2 to $4 \mu \mathrm{g} / \mathrm{kg}$ via a mucosal atomiser device. Clinical parameters such as heart rate, blood pressure, pulse oxygen saturation were monitored and recorded by the sedation nurse every 5 minutes after administration of sedatives.

Onset of sedation was defined as the time taken for patients to reach modified Ramsay sedation scale of $>3$ and was recorded (Table 1). Successful use of IN dexmedetomidine was defined by completion of procedure or investigation with dexmedetomidine as the only agent. When the patient required additional sedatives during the procedure, IV sedation would be administered by the attending physician. This would be counted as an unsuccessful event. No top up doses of IN dexmedetomidine were given to patients as top-up effects were deemed to be too gradual for patients waking up mid-procedure or investigation. Duration of sedation was defined as time from patients reaching modified Ramsay sedation scale of $>3$ to time of them waking up from sedation, which is defined as spontaneous opening of eyes without additional stimuli.

Any desaturation, bradycardia and hemodynamic instabilities were classified as adverse events. These were recorded by the attending physician and the specialist nurse. Desaturation was defined as pulse oximetry reading of $<95 \%$. Bradycardia was defined as $<60$ beats per minute. Haemodynamic instability was defined as systolic blood pressure less than $70 \mathrm{mmHg}+(2 \times$ age in years $)$ for children 1 to 10 years as per 2010 American Heart Association Guidelines for Cardiopulmonary Resuscitation and Emergency Cardiovascular Care (13).

Patients were monitored until they are fully awake and able to feed and drink before their discharge from sedation. Clinical follow-up was up to the point of discharge from sedation. The primary outcome was successful completion of procedures and investigations using only IN dexmedetomidine. Adverse events such as bradycardia, 
hypotension, and desaturation requiring intervention were also recorded.

We categorized the various procedures into "long" or "short" procedures according to the time taken for their completion. A "short" procedure was defined as a procedure or investigation that could be completed in less than 60 minutes. Procedures that take 60 minutes or longer were classified as "long". This cut-off was a local institutional definition. Hence, in this study, CT scans were classified as "short" procedures whereas AABR, dimercaptosuccinic acid (DMSA), mercaptoacetyltriglycine-3 (MAG3) and MRI scans were classified as "long". The initial dose of IN dexmedetomidine used was also divided into two categories - those who received $<3 \mu \mathrm{g} / \mathrm{kg}$ and those who received $\geq 3 \mu \mathrm{g} / \mathrm{kg}$ of IN dexmedetomidine. This cut off was chosen as prior studies had shown that a dose of $3 \mu \mathrm{g} / \mathrm{kg}$ was able to achieve adequate sedation for various procedures such as AABR and MRI scans $(7,14)$.

All paediatric patients who underwent sedation with IN dexmedetomidine and satisfied inclusion criteria were included. Sedation protocol was ratified by the sedation team and strictly adhered to for all procedures. Electronic medical record for all included patients were accessed. Data was collected and reviewed by two physicians to ensure accuracy. Retrospective review of outcomes and analysis of data was by independent parties.

The patients were grouped by whether they had successfully completed their procedures or investigations. We then compared the two groups and evaluated underlying factors associated with their outcomes.

\section{Statistical analysis}

Statistical analyses were performed with StataCorp software version 16.0 (StataCorp, Texas, USA). Descriptive data were presented with continuous data summarised as mean \pm standard deviation (SD), and categorical data shown as percentages. Analyses of onset of sedation and total duration of sedation were only done for cases successfully sedated by IN dexmedetomidine. Student's $t$-test was used to compare continuous variables between groups. Wilcoxon rank sum test was used for comparison of continuous variables that were not nominally distributed. Pearson chi square and Fisher's exact test were used for categorical variables. Univariate and multivariate logistic regression were used to analyse the clinically relevant variables such as age, weight, gender, ethnicity, type of procedures and dose of IN dexmedetomidine between the two groups. All statistical tests were performed at $5 \%$ level of significance.

\section{Ethical statement}

The study was conducted in accordance with the Declaration of Helsinki (as revised in 2013). The study was approved by the Domain Specific Review Board of National Health Group, Singapore (Study Reference Number 2020/00350) and individual consent for this retrospective analysis was waived.

\section{Results}

Hundred and five patients received IN dexmedetomidine for procedural sedation during the study period and all were included in the analysis. No patients were excluded for incomplete data. Baseline demographics of the patients who were successfully sedated and those who were not differed significantly in terms of median age and weight. Those who were successfully sedated with IN dexmedetomidine had median age of 24.5 months and median weight of $11.6 \mathrm{~kg}$. This was significantly different from those who failed sedation with IN dexmedetomidine alone, who had median age of 13.0 months and median weight of $9.9 \mathrm{~kg}(\mathrm{P}=0.024$ and 0.014 respectively). The mean onset of sedation did not differ significantly between the group that was successfully sedated and those who failed sedation $(14.9 \pm 7.7$ and $16.2 \pm 5.7$ minutes respectively, $\mathrm{P}=0.366)$. The former group had a longer duration of sedation $(107.2 \pm 64.6$ minutes $)$ than the latter group $(60.3 \pm 40.8$ minutes, $\mathrm{P}<0.001)$ (Table 2$)$.

The main indications for the use of IN dexmedetomidine were AABR (12, 11.4\%), CT (30, 28.6\%), and MRI (56, $53.3 \%)$ scans. Other indications were DMSA scan and renal MAG3 scan. Sixty (57.1\%) patients were successfully sedated using IN dexmedetomidine as a sole agent (Table 2). Duration of the procedure had significant correlation with sedation success $(\mathrm{P}=0.001)$. Forty patients $(88.9 \%)$ who failed had long procedures, 5 (11.1\%) had short procedures.

When analysed by indication, AABR, CT scans, DMSA, and MAG3 scans had success rates of $75.0 \%$ and above, as compared to MRI scans, which only had a success rate of $33.9 \%$. Baseline characteristics were not significantly different between different indications (Table 3).

A dose of $\geq 3 \mu \mathrm{g} / \mathrm{kg}$ of IN dexmedetomidine had an unadjusted odds ratio of 2.36 (95\% CI: 0.87-6.39; $\mathrm{P}=0.0865$ ) for successful sedation as compared to a reference of $<3 \mu \mathrm{g} / \mathrm{kg}$ but was not statistically significant. "Short" procedures were associated with an unadjusted odds ratio of 
Table 2 Baseline demographics and success of sedation

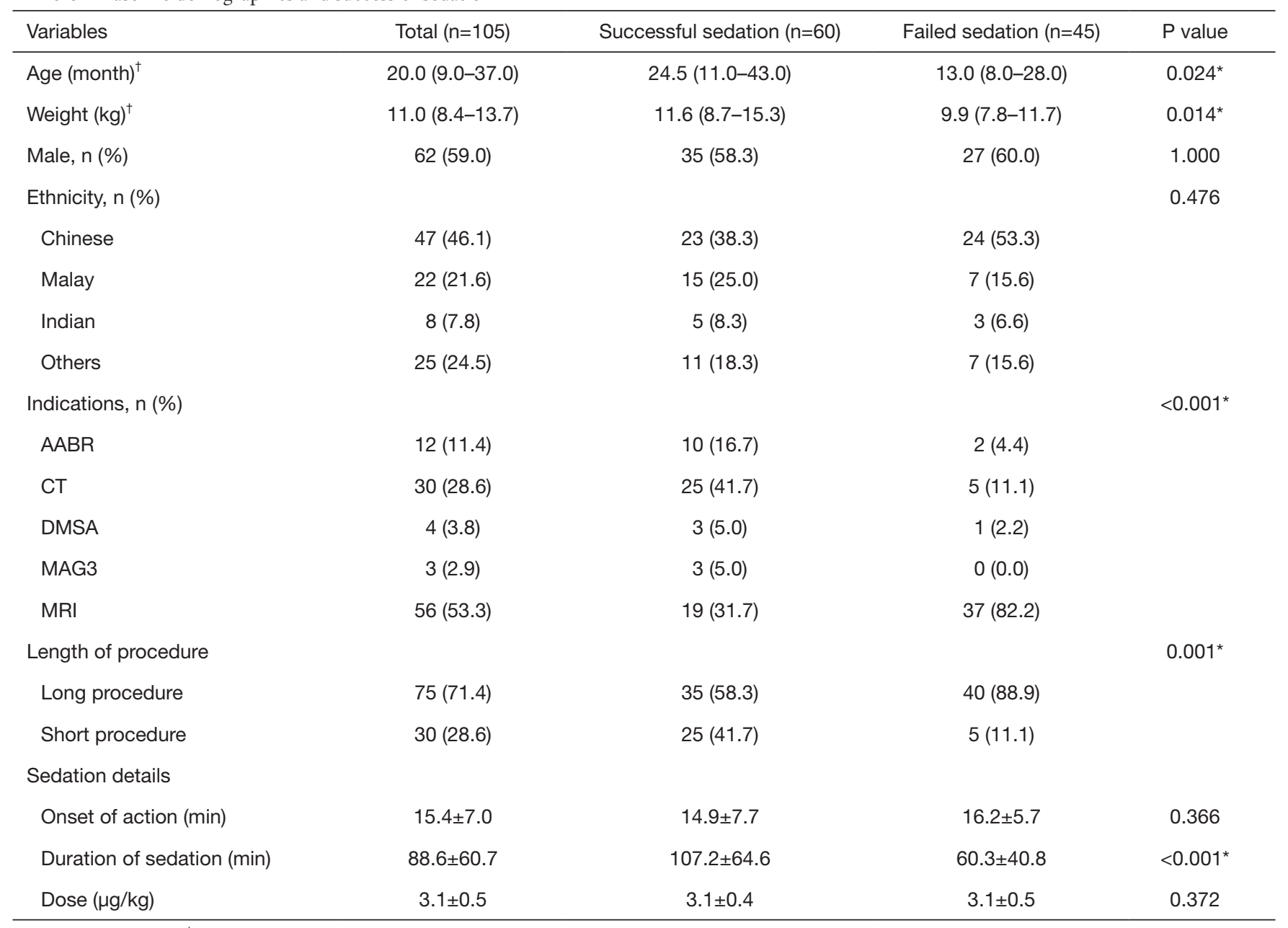

*, $\mathrm{P}$ value $<0.05 ;{ }^{\dagger}$, median (interquartile range). AABR, automated auditory brainstem response; CT, computerized tomography; DMSA, dimercaptosuccinic acid renal scan; MAG3, mercaptoacetyltriglycine 3 renogram; MRI, magnetic resonance imaging.

Table 3 Characteristics of patients and indications for IN dexmedetomidine use

\begin{tabular}{|c|c|c|c|c|c|c|}
\hline Variables & $\operatorname{AABR}(n=12)$ & $\mathrm{CT}(\mathrm{n}=30)$ & DMSA $(n=4)$ & MAG3 $(n=3)$ & MRI $(n=56)$ & $P$ value \\
\hline Weight $(\mathrm{kg})^{\dagger}$ & $9.2(8.4-10.8)$ & $11.8(9.9-14.0)$ & $10.8(9.5-12.3)$ & $16.7(7.8-19.7)$ & $10.5(7.8-13.9)$ & 0.479 \\
\hline Male, n (\%) & 7 (58.3) & $17(56.7)$ & $1(25.0)$ & $3(100.0)$ & $34(60.7)$ & 0.650 \\
\hline Sedation success, n (\%) & $10(83.3)$ & 25 (83.3) & $3(75.0)$ & $3(100.0)$ & 19 (33.9) & $<0.001^{\star}$ \\
\hline
\end{tabular}

${ }^{*}$, $\mathrm{P}$ value $<0.05{ }^{\dagger}$, median (interquartile range). AABR, automated auditory brainstem response; CT, computerized tomography; DMSA, dimercaptosuccinic acid renal scan; MAG3, mercaptoacetyltriglycine 3 renogram; MRI, Magnetic resonance imaging.

5.71 (95\% CI: $1.98-16.53$; $\mathrm{P}<0.001)$ for successful sedation. Age and weight had unadjusted odds ratio of $1.01(95 \% \mathrm{CI}$ : $1.00-1.02 ; \mathrm{P}=0.211)$ and 1.06 (95\% CI: $0.99-1.14 ; \mathrm{P}=0.066)$ respectively for successful use of IN dexmedetomidine which were not statistically significant. The odds ratio for male gender and different ethnicities were also not statistically significant.

On multivariate analysis, "short" procedures remained 
Table 4 Multivariate analysis for prediction of successful use of IN dexmedetomidine

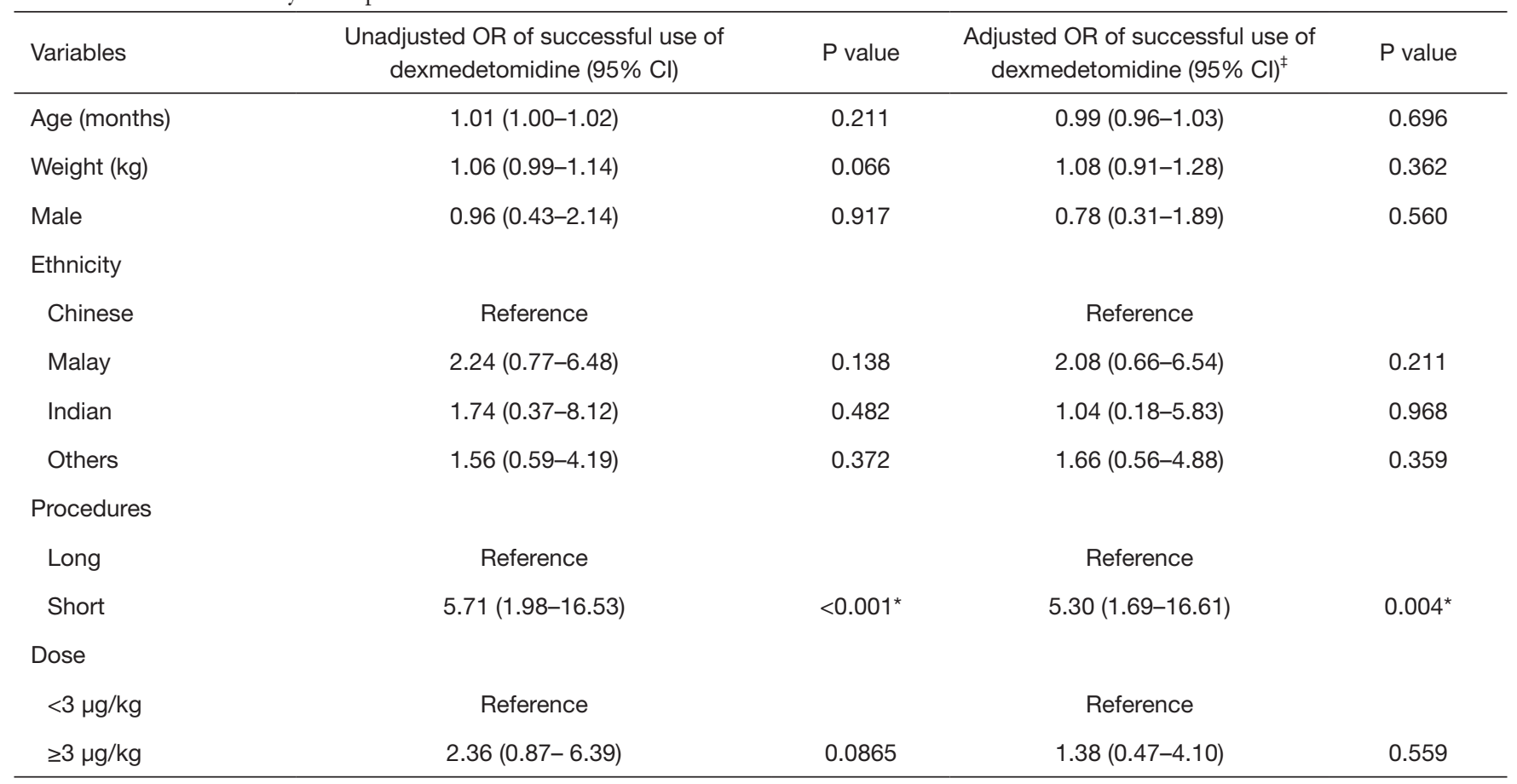

*, P value $<0.05$; ${ }^{\ddagger}$, adjusted for age, weight, gender, ethnicity, dose category of IN dexmedetomidine, length of procedure. IN, intranasal; OR, odds ratio; $\mathrm{Cl}$, confidence interval.

significantly associated with achieving successful sedation, adjusted odds ratio 5.30 (95\% CI: 1.69-16.61; $\mathrm{P}=0.004$ ) whereas other variables such as age, weight, gender, ethnicity and dose did not (Table 4).

No adverse events were reported throughout the study period.

\section{Discussion}

With rising concern about ICU delirium and neurotoxicity effects of various sedatives on the developing brain, dexmedetomidine has become the preferred choice of sedative in the ICU, given its reported neuroprotective effect (15-22). This is in contrast to procedural sedation where the use of dexmedetomidine is not routine. Its sedation effectiveness when delivered intranasally, was reported to be comparable to various other agents such as IN ketamine, midazolam and oral chloral hydrate $(3,23,24)$. An IN route of administration is thought to be more convenient as compared to IV sedation as it does not require for placement of IV cannulas. Pharmacological bioavailability—and, hence, clinical efficacy-may be more consistent than oral sedatives as the latter may be regurgitated or rejected by younger patients. However, the onset and duration of action may vary between individuals. It was thus important to characterise a patient population that would best benefit from the use of IN dexmedetomidine.

In our study, the overall success rate of IN dexmedetomidine was lower than other studies. There were several reasons for this observation. Firstly, we had defined successful use of dexmedetomidine as completion of investigations with this agent solely. This definition was chosen because it was the most clinically relevant end point and reflects accurately the pragmatic real-world use of IN dexmedetomidine. Other studies that had reported their successful use had different endpoints such as its ability to achieve adequate sedation scores, and satisfactory parental separation $(3,5)$. These endpoints are easier to achieve and would inflate estimates of sedation success.

It was notable that patients who had successful sedation using IN dexmedetomidine and those who did not, differed significantly in their age, weight and underlying indications for sedation $(\mathrm{P}=0.024,0.014,<0.001$ respectively). The median age and weight for the successfully sedated group were higher than the other group. This finding differed 
from existing literature which suggested that children under three years, dose per body weight of IN dexmedetomidine required to achieve adequate sedation was higher in those who were older (25). One possible explanation could be compliance to sleep deprivation, which was routinely instructed to the parents prior to procedure sedation, was higher for older children than younger. Nonetheless, when adjusted for other factors such as gender, ethnicity, length of procedure and dose of IN dexmedetomidine, they were insignificant in influencing the outcome.

In the successful sedation group, there were fewer MRI scans $(31.7 \%$ versus $82.2 \%)$ and a greater composition of AABR (16.7\% versus $4.4 \%)$ and CT scans $(41.7 \%$ versus $11.1 \%$ ) (Table 2). This suggests that the most important contributor to sedation success was the selection of an appropriate procedure.

On multivariate analysis, short procedures were shown to have an adjusted odds ratio of 5.30 (95\% CI: 1.69-16.61; $\mathrm{P}=0.004$ ) for successful sedation using IN dexmedetomidine alone when compared against long procedures. The outcomes for short procedures are congruous with previously published results, whereas the success rate for long procedures are markedly lower (5-7). On further analysis, it was apparent that procedures such as AABR, CT, DMSA, MAG3 had a much higher success rate of at least $75 \%$, which is comparable to published reports. In contrast, success rate of MRI scans was only $33.9 \%$. This could suggest that poor success rates for long procedures were driven by failures during MRI scans rather than being due to the length of the procedure alone.

The lower success rate in patients undergoing MRI could be attributable to several reasons. In our centre, delays in schedules were more likely to occur for MRI scans than other radiological investigations. This may result in a prolonged waiting time and, hence, lengthen the duration needed for the patient to remain sedated. Patients would also need to be shifted from ward bed to MRI trolley, ear plugs and a head cap would need to be placed; these movements could contribute to the patient waking up from sedation. As per center protocol, no top up dose was administered during the sedation process. This is because of the gradual onset of action, limiting its efficacy as a rescue sedative.

In addition, a starting dose of $3 \mu \mathrm{g} / \mathrm{kg}$ was used in many of our patients who had failed to complete MRI scans with IN dexmedetomidine as a sole agent. This was lower than what was used in prior studies, which had shown that a higher dose of $4 \mu \mathrm{g} / \mathrm{kg}$ was associated with better outcomes and lower rate of anaesthetic rescue $(4,26)$. This suggests that long and rest-disruptive procedures-in particular MRI scans-should be performed with a higher dose of sedation.

From our observation and prior studies, sedation induced by IN dexmedetomidine is similar to natural sleep $(1,27)$. It is often light at the beginning and deeper as time goes by. Hence, patients may still wake up easily from movement in the initial phases corresponding to sedation level of modified Ramsay score 3. If this coincides with the need to move the patient, an additional small dose of IV propofol is often needed. A dose of $1-2 \mathrm{mg} / \mathrm{kg}$ of IV propofol could help to re-induce sleep for these patients and allow them to complete the scan without any additional sedatives. Out of 37 patients who failed to use IN dexmedetomidine as the solo agent for their MRI scans, 7 (18.9\%) of them had completed the scan with this small bolus dose of propofol. This may also suggest that sufficient time should be given prior to procedures when using IN dexmedetomidine to allow for adequate depth of sedation.

This study was not powered to look at potential side effects of IN dexmedetomidine and this was not investigated.

\section{Study limitations}

This was a retrospective cohort study with a limited sample size which would impact on statistical power. Additionally, IN dexmedetomidine has a gradual onset of action which may vary between individuals. This makes accuracy of timing of scans an important factor that can affect its efficacy. Sedation practices may vary between centres, limiting the generalisability of the above results. Lastly, the choice of IN dexmedetomidine as initial sedation was left to the primary physician's own discretion and comfort, rendering the study at risk of selection bias.

\section{Conclusions}

IN dexmedetomidine is effective for procedural sedation for paediatric patients. The most important predictor for sedation success was indication for sedation; long procedures such as MRI scans were associated with poorer results. The study is hypothesis-generating and more research into this field is necessary.

\section{Acknowledgments}

The authors would like to thank Ms Modesto Vanessa 
Iturriaga, Ms Gan Ling Ling Susanna for their contribution to data collection, and all staff of division of critical care medicine, Department of Paediatrics, National university Hospital for their support.

Funding: None.

\section{Footnote}

Reporting Checklist: The authors have completed the STROBE checklist. Available at http://dx.doi.org/10.21037/ tp-20-358

Data Sharing Statement: Available at http://dx.doi. org/10.21037/tp-20-358

Peer Review File: Available at http://dx.doi.org/10.21037/tp20-358

Conflicts of Interest: All authors have completed the ICMJE uniform disclosure form (available at: http://dx.doi. org/10.21037/tp-20-358). The authors have no conflicts of interest to declare.

Ethical Statement: The authors are accountable for all aspects of the work in ensuring that questions related to the accuracy or integrity of any part of the work are appropriately investigated and resolved. The study was conducted in accordance with the Declaration of Helsinki (as revised in 2013). The study was approved by the Domain Specific Review Board of National Health Group, Singapore (Study Reference Number 2020/00350) and individual consent for this retrospective analysis was waived.

Open Access Statement: This is an Open Access article distributed in accordance with the Creative Commons Attribution-NonCommercial-NoDerivs 4.0 International License (CC BY-NC-ND 4.0), which permits the noncommercial replication and distribution of the article with the strict proviso that no changes or edits are made and the original work is properly cited (including links to both the formal publication through the relevant DOI and the license). See: https://creativecommons.org/licenses/by-nc-nd/4.0/.

\section{References}

1. Hayashi Y, Maze M. Alpha 2 adrenoceptor agonists and anaesthesia. Br J Anaesth 1993;71:108-18.

2. Uusalo P, Lehtinen M, Loyttyniemi E, et al. Premedication with intranasal dexmedetomidine decreases barbiturate requirement in pediatric patients sedated for magnetic resonance imaging: a retrospective study. BMC Anesthesiol 2019;19:22.

3. Gupta A, Dalvi NP, Tendolkar BA. Comparison between intranasal dexmedetomidine and intranasal midazolam as premedication for brain magnetic resonance imaging in pediatric patients: A prospective randomized double blind trial. J Anaesthesiol Clin Pharmacol 2017;33:236-40.

4. Olgun G, Ali MH. Use of Intranasal Dexmedetomidine as a Solo Sedative for MRI of Infants. Hosp Pediatr 2018. [Epub ahead of print]. doi: 10.1542/hpeds.2017-0120.

5. Ghai B, Jain K, Saxena AK, et al. Comparison of oral midazolam with intranasal dexmedetomidine premedication for children undergoing CT imaging: a randomized, double-blind, and controlled study. Paediatr Anaesth 2017;27:37-44.

6. Reynolds J, Rogers A, Capehart S, et al. Retrospective Comparison of Intranasal Dexmedetomidine and Oral Chloral Hydrate for Sedated Auditory Brainstem Response Exams. Hosp Pediatr 2016;6:166-71.

7. Reynolds J, Rogers A, Medellin E, et al. A prospective, randomized, double-blind trial of intranasal dexmedetomidine and oral chloral hydrate for sedated auditory brainstem response (ABR) testing. Paediatr Anaesth 2016;26:286-93.

8. Haselkorn T, Whittemore AS, Udaltsova N, et al. Shortterm chloral hydrate administration and cancer in humans. Drug Saf 2006;29:67-77.

9. Cozzi G, Norbedo S, Barbi E. Intranasal Dexmedetomidine for Procedural Sedation in Children, a Suitable Alternative to Chloral Hydrate. Paediatr Drugs 2017;19:107-11.

10. Kamat PP, Kudchadkar SR, Simon HK. Sedative and Anesthetic Neurotoxicity in Infants and Young Children: Not Just an Operating Room Concern. J Pediatr 2019;204:285-90.

11. Wang Y, Han R, Zuo Z. Dexmedetomidine post-treatment induces neuroprotection via activation of extracellular signal-regulated kinase in rats with subarachnoid haemorrhage. Br J Anaesth 2016;116:384-92.

12. Li F, Wang X, Deng Z, et al. Dexmedetomidine reduces oxidative stress and provides neuroprotection in a model of traumatic brain injury via the PGC- $1 \alpha$ signaling pathway. Neuropeptides 2018;72:58-64.

13. Kleinman ME, Chameides L, Schexnayder SM, et al. Part 14: pediatric advanced life support: 2010 American Heart Association Guidelines for Cardiopulmonary Resuscitation 
and Emergency Cardiovascular Care. Circulation 2010;122:S876-908.

14. Behrle N, Birisci E, Anderson J, et al. Intranasal Dexmedetomidine as a Sedative for Pediatric Procedural Sedation. J Pediatr Pharmacol Ther 2017;22:4-8.

15. Scott-Warren VL, Sebastian J. Dexmedetomidine: its use in intensive care medicine and anaesthesia. BJA Education 2016;16:242-6.

16. Sun R, Wang S, Li S, et al. Effects of dexmedetomidine on delirium and mortality during sedation in ICU patients: a systematic review and meta-analysis protocol. BMJ Open 2019;9:e025850.

17. Loepke AW. Developmental neurotoxicity of sedatives and anesthetics: a concern for neonatal and pediatric critical care medicine? Pediatr Crit Care Med 2010;11:217-26.

18. Young C, Jevtovic-Todorovic V, Qin YQ, et al. Potential of ketamine and midazolam, individually or in combination, to induce apoptotic neurodegeneration in the infant mouse brain. Br J Pharmacol 2005;146:189-97.

19. Brambrink AM, Evers AS, Avidan MS, et al. Ketamineinduced neuroapoptosis in the fetal and neonatal rhesus macaque brain. Anesthesiology 2012;116:372-84.

20. Tan X, Zeng Y, Tu Z, et al. TRPV1 Contributes to the Neuroprotective Effect of Dexmedetomidine in Pilocarpine-Induced Status Epilepticus Juvenile Rats. Biomed Res Int 2020;2020:7623635.

21. Li F, Wang X, Zhang Z, et al. Dexmedetomidine Attenuates Neuroinflammatory-Induced Apoptosis after Traumatic Brain Injury via Nrf2 signaling pathway. Ann

Cite this article as: Fan L, Lim Y, Wong GS, Taylor R. Factors affecting successful use of intranasal dexmedetomidine: a cohort study from a national paediatrics tertiary centre. Transl Pediatr 2021;10(4):765-772. doi: 10.21037/tp-20-358
Clin Transl Neurol 2019;6:1825-35.

22. Wang L, Liu W, Zhang Y, et al. Dexmedetomidine had neuroprotective effects on hippocampal neuronal cells via targeting lncRNA SHNG16 mediated microRNA-10b-5p/ BDNF axis. Mol Cell Biochem 2020;469:41-51.

23. Neville DN, Hayes KR, Ivan Y, et al. Doubleblind Randomized Controlled Trial of Intranasal Dexmedetomidine Versus Intranasal Midazolam as Anxiolysis Prior to Pediatric Laceration Repair in the Emergency Department. Acad Emerg Med 2016;23:910-7.

24. Ibrahim M. A prospective, randomized, double blinded comparison of intranasal dexmedetomodine vs intranasal ketamine in combination with intravenous midazolam for procedural sedation in school aged children undergoing MRI. Anesth Essays Res 2014;8:179-86.

25. Yu Q, Liu Y, Sun M, et al. Median effective dose of intranasal dexmedetomidine sedation for transthoracic echocardiography in pediatric patients with noncyanotic congenital heart disease: An up-and-down sequential allocation trial. Paediatr Anaesth 2017;27:1108-14.

26. Tug A, Hanci A, Turk HS, et al. Comparison of Two Different Intranasal Doses of Dexmedetomidine in Children for Magnetic Resonance Imaging Sedation. Paediatr Drugs 2015;17:479-85.

27. Miller J, Xue B, Hossain M, et al. Comparison of dexmedetomidine and chloral hydrate sedation for transthoracic echocardiography in infants and toddlers: a randomized clinical trial. Paediatr Anaesth 2016;26:266-72. 